\title{
P 024 THE EMOTIONAL RESPONSES OF STAFF TO MOVING TO A NEW HOSPICE BUILDING
}

Jane Murray, Kathy Armour, Chantal Meystre. Marie Curie Cancer Care, Solihull, UK

10.1136/bmjspcare-2014-000654.65

Background Work relocation is a major change and stressful, not only due to change in layout and functional delivery (Peach, 2005) but particularly, in palliative care, where memories are attached to the original worksite (Murphy, 2012). Research applying attachment theory links places to identities, giving insight to the stress and loss of moving (Speller, 2000). Our hospice was relocating nearby to a bespoke unit from a building occupied for nearly 50 years. Project planning managed practical issues but psychological and emotional aspects of the move were undefined.

Aim To explore staff and volunteers experiences of moving at the time of a hospice relocation.

Methods All hospice staff were eligible for the study and invited to anonymously complete a modified adult attachment questionnaire (Brennan, 1998), with additional quantitative and semi-structured qualitative elements, in the week prior to relocating. Staff views were triangulated with returns from volunteers. As no conflicting themes emerged they are reported together.

Results 29 clinical staff, 5 non-clinical staff and 8 volunteers participated (31\% return rate). Their average length of service was 6.2 years ranging from $<1-23$ years. Thematic analysis revealed major themes around memories and remembrance; atmosphere created by caring staff and volunteers; experiences with patients, families and colleagues; and a positivity regarding caring in the new environment. Some staff were experiencing anticipatory grief and some required ways of ending an era.

Conclusion The study captured a unique exploration of staff emotional reactions to relocating. It demonstrated that anxiety was related to an unconscious anticipatory grief linked to memories and experiences within the existing hospice, not an attachment to the building as the quantitative results (Meystre, 2013) suggested. This realisation demonstrates the power of mixed method studies to investigate complex phenomena within palliative care. 